\title{
Vegetation Mapping of a Tomato Crop using Multilayer Perceptron (MLP) Neural Network in Images Acquired by Remote Sensing from a UAV
}

\author{
Ramesh Kestur \\ Department of Electronics \& Instrumentation \\ Bangalore Institute of Technology, \\ Bangalore, 560004, \\ Karnataka, India
}

\author{
Meenavathi M. B. \\ Department of Electronics \& Instrumentation, \\ Bangalore Institute of Technology, \\ Bangalore, 560004, \\ Karnataka, India
}

\begin{abstract}
Remote sensing from an Unmanned Aerial Vehicle (UAV), also known as Low Altitude Remote Sensing provides interesting options for applications in agriculture. Vegetation mapping is an important application in remote sensing applications. In this work vegetation mapping is carried out in a tomato crop. Aerial imagery of tomato crop is acquired by a Quadcoptor UAV with an optical sensor as the payload. The optical sensor is the camera module of a Raspberry PI single board Single Board Computer (SBC). Vegetation mapping of the tomato crop is carried out by segmentation of the tomato crop images using the proposed MLP-SEG method. Performance of MLP-SEG method is compared with a Support Vector Machine (SVM) based method SVM-SEG. Confusion matrix parameters are used to analyse the performance of the proposed method. The results indicate that MLP-SEG performance is comparable to SVM-SEG.
\end{abstract}

\section{General Terms}

Vegetation mapping, Unmanned Aerial Vehicle (UAV), Low Altitude Remote Sensing (LARS),

\section{Keywords}

Support Vector Machine Segmentation (SVM-SEG), Multi Layer Perceptron Segmentation (MLP-SEG).

\section{INTRODUCTION}

Remote sensing from satellite and manned aircrafts has made invaluable contributions to a wide range of applications in agriculture such as crop type classification , crop monitoring and crop yield estimation[1][2][3]. Large swathes of land area covering several kilometers are analyzed with a range of imagery such as multispectral and hyperspectral imagery. The satellite remote sensing resolution has medium resolution $(\sim$ 5 to 30Metres) from sensors such as LANDSAT, SPOT and ETM. Agriculture applications of satellite and manned aircraft based remote sensing is however at a macro level where studies are carried out at village, town and cities covering several kilometers in area.

In recent years there is an increased proliferation of Unmanned Aerial systems which has provided an interesting remote sensing platform that complements the traditional satellite and manned aircraft based platforms. Remote sensing from an UAV, also known as Low Altitude Remote Sensing (LARS) [4][5] or near earth remote sensing offers several advantages. They are low cost, can be operated under cloud cover, provide high spatial resolution and high temporal resolution.. Further LARS offers several specific advantages in agriculture applications, particularly in Precision Agriculture (PA). Analysis can be carried out at micro level for site specific applications . Crops can be studied crops at a level of canopies, plants, fruits and flowers for studies such as pest identification and yield estimation.

PA applications depend on performing a vegetation segmentation process as a necessary and mandatory initial step which aims to detect vegetation area in agriculture field images. Such process could easily be performed using vegetation indices such as NDVI and SAVI, Weighted difference Vegetation index (WDVI) derived from multispectral imagery. However, although multi spectral and hyper spectral cameras are available on UAS platforms, they are not widely used due to issues with radiometric corrections and consistency of data acquired over multiple flights due to illumination issues. The large availability of UAV platforms with vision spectrum cameras or RGB cameras has explored the potential of low cost RGB sensors for vegetation monitoring [4]. However, developing vegetation segmentation techniques using RGB images is a challenging problem due to low spectral resolution. [6] analyzed UAV RGB images for vegetation segmentation. The RGB was transformed to HUE images. Thresholds were used on the HUE histograms to discriminate between vegetation and non vegetation regions. Thresholds were determined by fitting Gaussian curves in HUE histograms. [7] used indices derived from normalized RGB values to derive the Vegetation Fraction (VF) in early season wheat field for temporal study of wheat crops. In recent times classification based on machine learning is gaining wide popularity. Support Vector Machines (SVM) based classification is used widely in remote sensing of satellite imagery[8][9][10][11]. Further Neural Network based classification such as Multi Layer Perceptrons based classification is drawing research interest in vegetation mapping using RGB imagery.

In this work vegetation mapping of a tomato crop is realized by analyzing aerial imagery of tomato crops acquired by remote sensing (LARS) carried out using a Quadrotor UAV. Tomato crop area and non crop area are mapped by classification carried out using SVM and Multi Layer Perceptron (MLP) neural networks. Images acquired at varying altitudes are analyzed to validate the robustness of classification. Performance of crop area and non crop is analyzed using confusion matrix based parameters of Precision, Recall and Accuracy. The results demonstrate that MLP performs better than SVM.

\section{METHODOLOGY}

This section describes the proposed method MLP-SEG for segmentation of crop and non crop areas in the aerial images of tomato crops acquired by carrying out LARS from a quadcopter UAV. The performance of MLP-SEG is compared with crop and non crop classification using Support Vector Machines (SVM-SEG). Further, criteria for evaluation of 
performance of segmentation and the method of data acquisition is discussed.

\subsection{Multilayer Perceptron segmentation (MLP-SEG)}

An Artificial Neural Network (ANN) is a system of interconnected neurons that are used to model non linear relationships. A Multilayer Perceptron Neural network (MLP) [12] is a class of feed forward ANN. The MLP model consists of three types of layers, an input layer, one or more hidden layers and an output layer. The model of a single hidden layer MLP is shown in Figure 1.The weight of the hidden neuron is given by

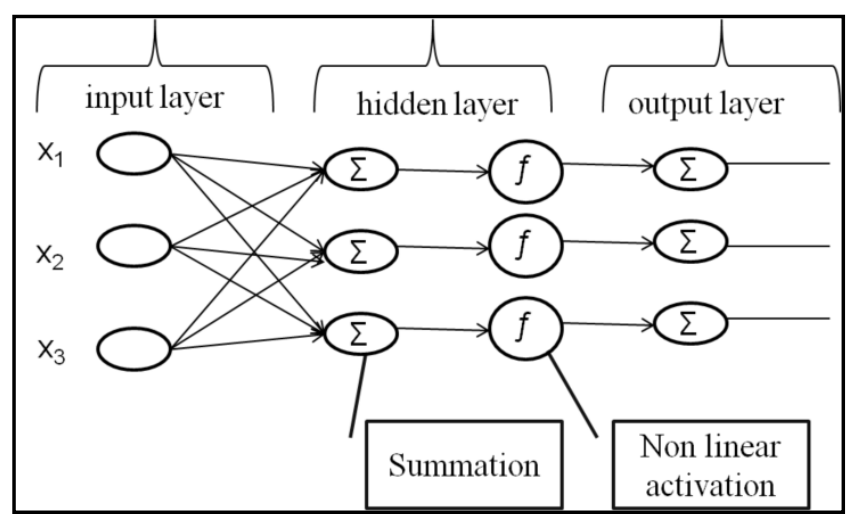

Figure 1: Multilayer Perceptron

$$
y_{j}=f\left(\sum_{i=1}^{n} w_{j i} x_{i}\right)
$$

The $j^{\text {th }}$ neuron $y_{j}$ is given by equation (1) where $f($.$) is an$ element wise non linear activation. The non linear activation is either a sigmoid function or a Rectified Linear Unit ( RELU) function, given by equation (2)

$$
f(k)=\max (0, k)
$$

The MLP network used in proposed MLP-SEG is shown in Figure 2. The input vector to the network is the R,G,B values of the pixel and the Hue, Saturation, Value (HSV) transformed values of the RGB pixel. The MLP network comprises of 5 hidden layers. The number of hidden neurons in each layer is depicted in Figure 2. Batch normalization with Stochastic Gradient Descent (SGD) and a momentum is used in the gradient descent for back propagation. Dropout regularization is used to avoid over fitting.

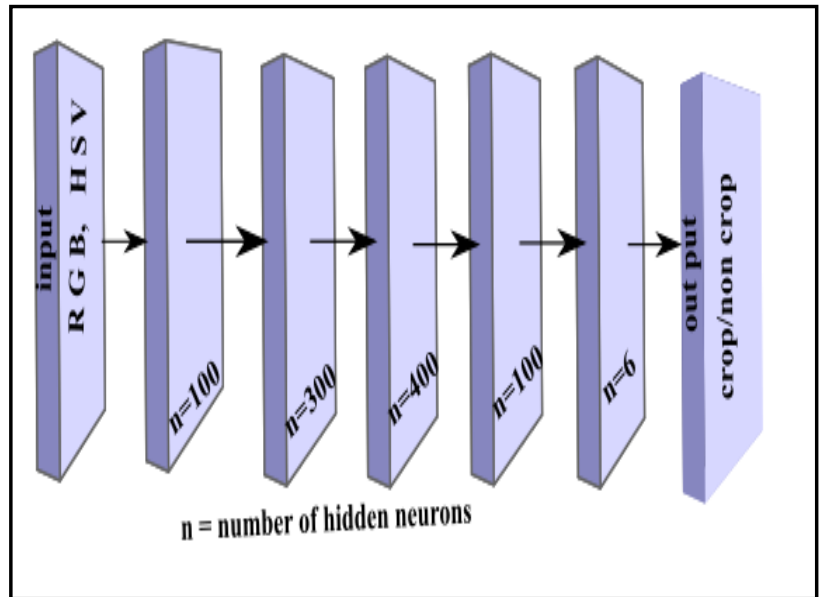

Figure 2: MLP network of proposed MLP-SEG

The hyper-parameters used in the MLP model is shown in Table 1

Table 1 : Hyper parameters of MLP network

\begin{tabular}{|l|l|}
\hline Parameters & Specification \\
\hline No of hidden layers & $5 \mathrm{~m}$ \\
\hline Batch size & 65536 \\
\hline No of epochs & 10000 \\
\hline momentum & 0.9 \\
\hline Dropout rate & 0,2 \\
\hline
\end{tabular}

The output of the MLP network is classified gray level image. The gray level image is converted into a binary image by gray level thresholding using OTSU [13] method.

\subsection{Support Vector Machine}

Support Vector Machine (SVM) [14] is a supervised algorithm popularly used for classification and regression. SVM transforms the input space to a non linear mapping function in the higher dimensional feature space. In this work, SVM is used for supervised binary classification (SVM-SEG)

$$
x_{i} \in \mathfrak{R}^{d}(i=1,2, \ldots, \mathrm{N}) .
$$

Where $x_{i}$ is the input training dataset with $\mathbf{N}$ vectors. For each $x_{i}$ a binary target $y_{i}$ is assigned. The data is mapped to a higher dimensional feature space $\Phi(X)$. The membership decision rule is based on the function $\operatorname{sign}[\mathrm{f}(\mathrm{x})]$ where $f(x)$ represents the discriminant function associated with the hyperplane and is given by

$$
f(x)=\mathrm{w}^{*} . \Phi(x)+b .
$$

Where $\mathrm{w}^{*}$ is the weight vector of the optimal hyperplane of the higher dimensional space. The optimized discrimination function is given by

$$
f(x)=\sum_{i \in S} \alpha_{i} y_{i} K\left(x_{i}, x\right)+b \ldots \ldots \ldots(5)
$$

where

$$
K\left(x_{i}, x\right)=\exp \left(-l\left\|x_{i}-x\right\|^{2}\right)
$$


Is the Radial Basis (RBF) kernel function and $\alpha_{i}$ are the support vectors and $l$ is a parameter that determines the width of the Gaussian kernel

\subsection{Performance analysis of proposed method.}

The performance of classification of vegetation and non vegetation areas is analysed using the confusion or the contingency matrix. The performance parameters precision, recall, F1-score and accuracy derived from the confusion matrix are analysed. Further visualization of the outputs for different images are provided that includes heatmaps.

\subsection{Data Acquisition.}

The data is acquired by quadcopter UAV flying over tomato farms with an optical payload sensor. The optical payload is the camera module of a Rasperry Pi single board computer (SBC). Aerial video of the tomato farm was acquired. Images were extracted from the video. Further images that had a good representation of the tomato crops was chosen for analysis.

\section{RESULTS AND DISCUSSION}

In this section the results from crop and non crop segmentation is discussed. The results of the segmentation using the proposed MLP-SEG and SVM-SEG is discussed. Performance of MLP-SEG is compared with ELM-SEG. Further, visualization of crop and non-crop segmentation is discussed. Figure shows the visual outputs of the images. Figure 5(a) is the original image. The results of segmentation from SVM-SEG is shown in Figure 5(b) . Figure 5(d) shows a heatmap of the grayscale values of the output of segmentation using MLP. The grayscale values are thresholded to a binary value using OTSU thresholding. The binary output is shown in Figure 5(c). From visual interpretation it can be observed that SVM-SEG has segmented more non crop area pixels as crop area pixels, thereby increasing the false positives. Further, the results of the segmentation of three other images out of the six analyzed images are depicted in Figure 6. Figure 6 (a)(d) (g) are the original images. The images are acquired at different altitudes. Figure $6(\mathrm{c})(\mathrm{e})(\mathrm{h})$ are the outputs of MLP-SEG and the Figure 6(c) (f)(i) are the heatmaps of the gray scale outputs of segmentation using MLP.

A total of six image acquired at varying altitudes was analyzed to validate the robustness of the proposed method. The precision and recall performance of the six images is shown in Figure 4. It can be observed that MLP-SEG performs better than SVM-SEG on both precision and recall performance consistently for all the six images. Figure 3 shows a box plot of the performance of MLP-SEG and SVM$\mathrm{SE}$ on the precision, recall, F1-score and Accuracy parameters for the six images. It can be observed that MLP-SEG performs better than SVM-SEG for the Precision, F1-score and accuracy parameters. However, SVM-SEG shows better performance on recall parameter. This is because SVM the more number of false positives in SVM-SEG. This can also be observed in the visualization of segmentation results in Figure

Comparison of SVM and Multi-layer Perceptron models

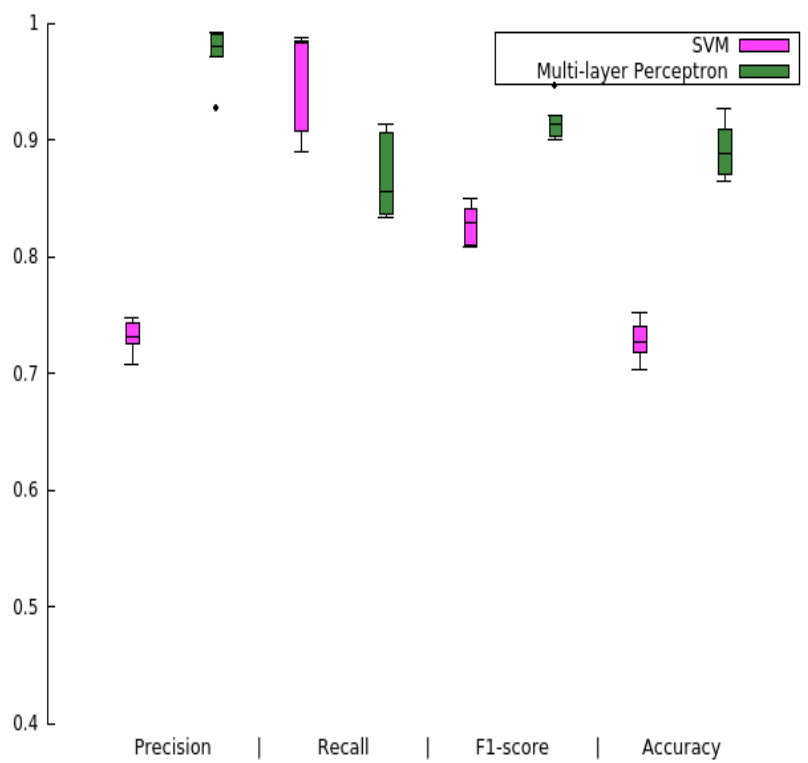

Figure 3: Performance parameters comparison of MLPSEG and SVM-SEG

Precision v/s Recall

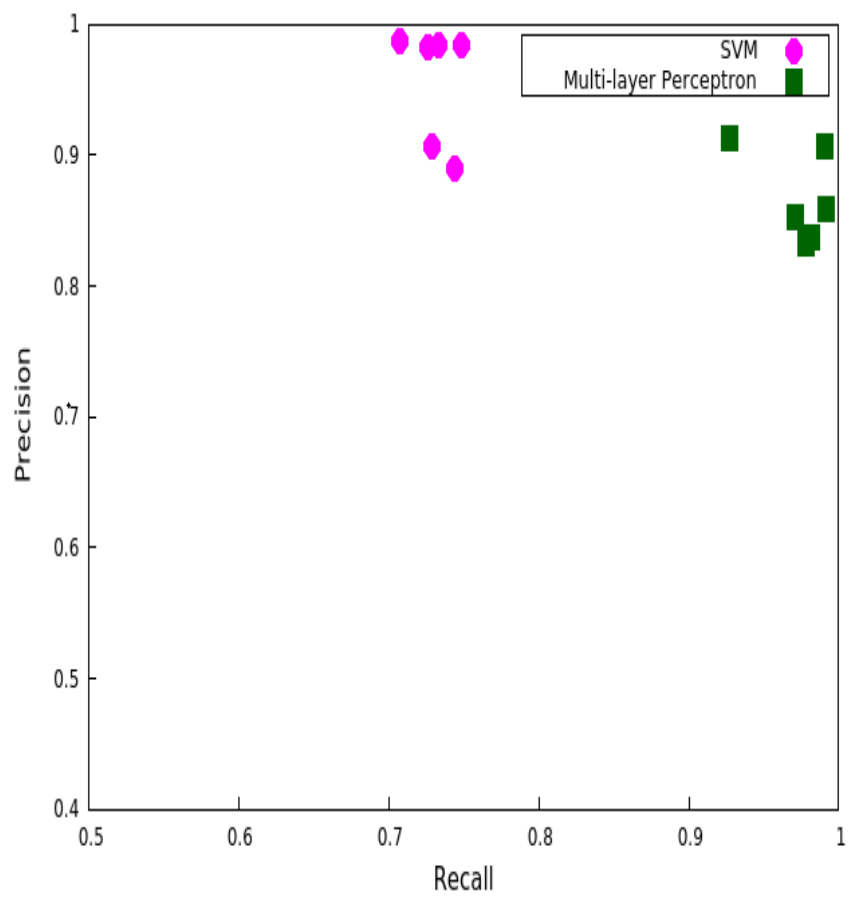

Figure 4: Precision recall performance 

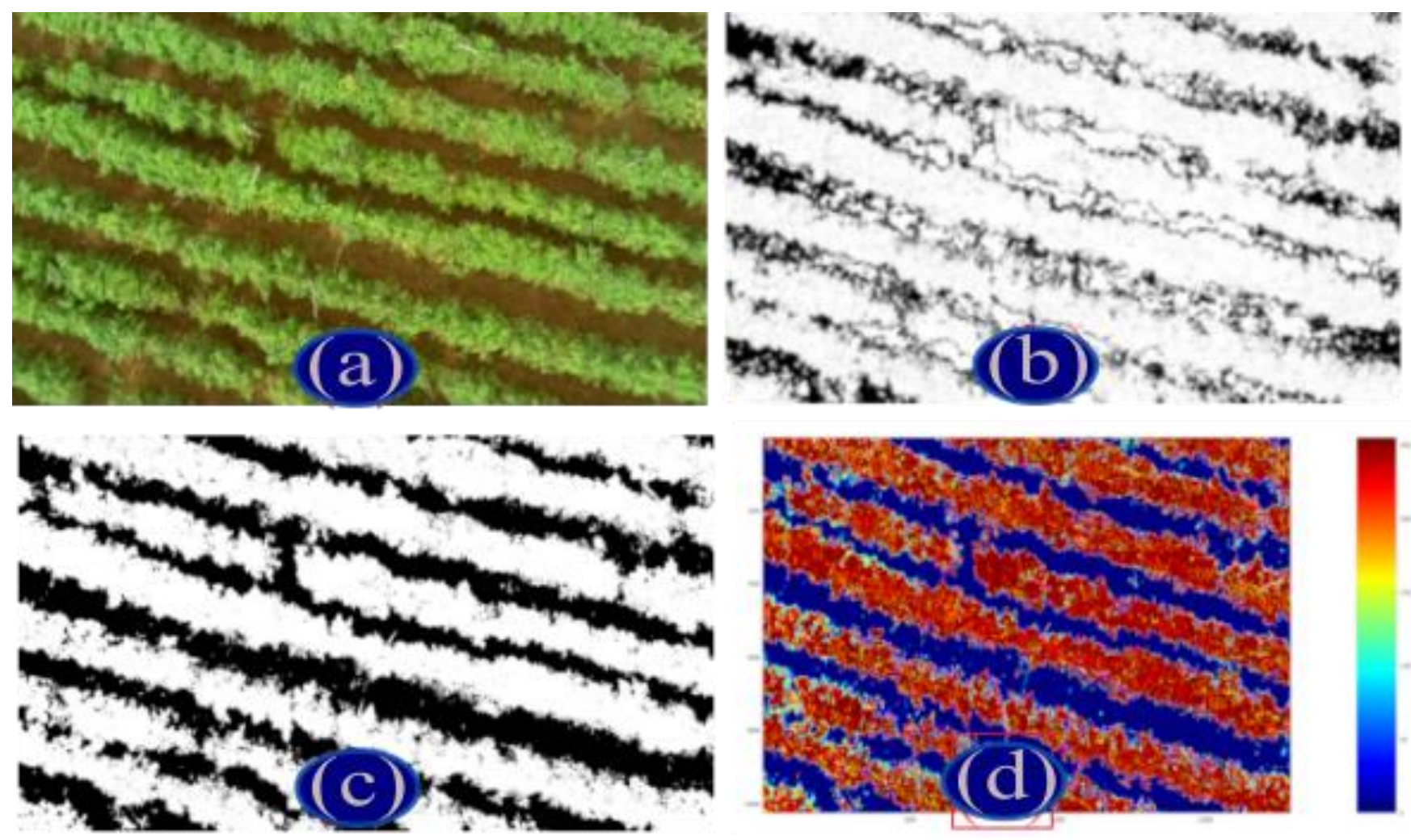

Figure 5: Visualization of proposed method
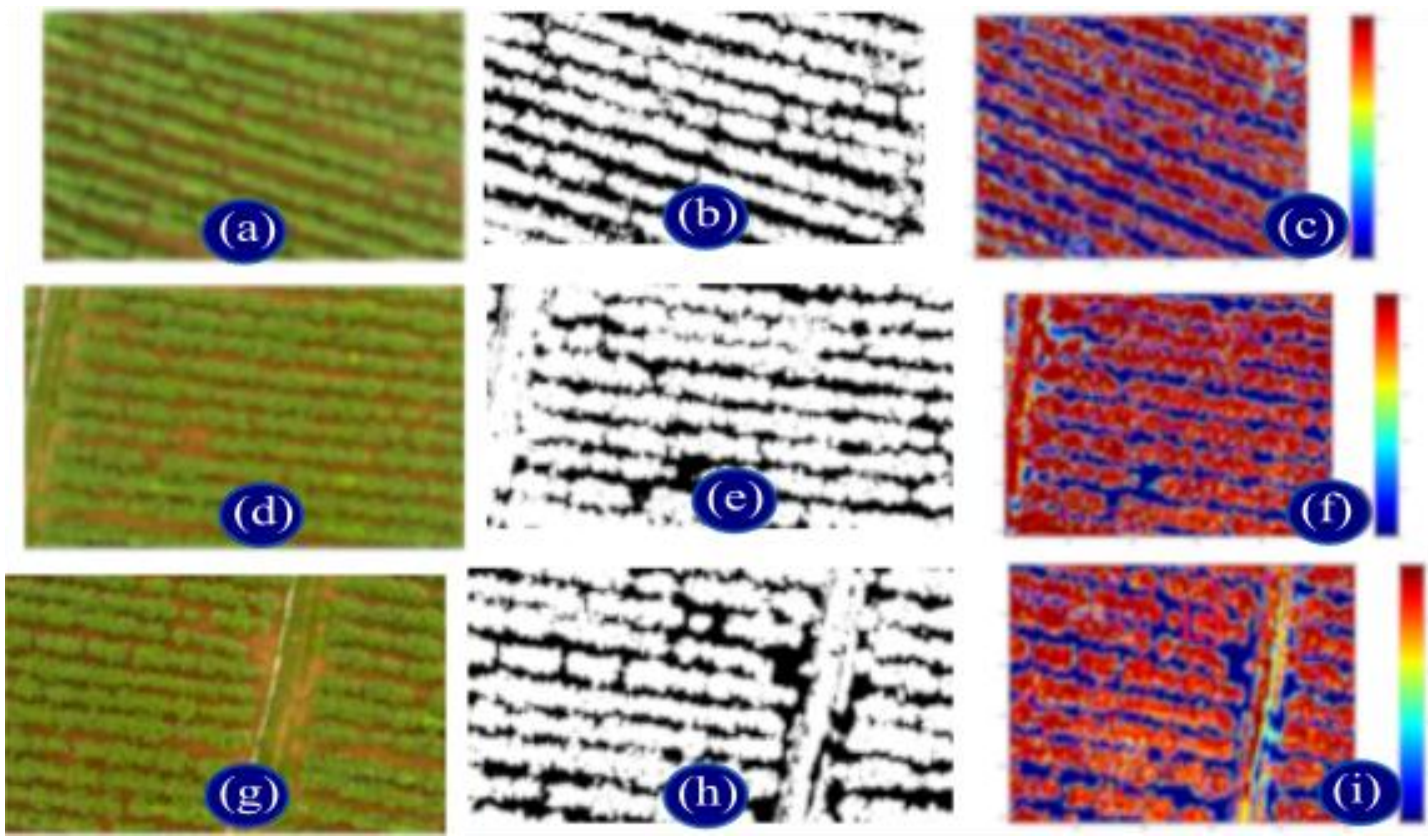

Figure 6: Visualization of results of three different images

\section{CONCLUSION}

Vegetation mapping of tomato crop was successfully carried using LARS. Segmentation of crop and non crop area was realized using the proposed method MLP-SEG. Performance was compared with SVM-SEG. The performance of proposed MLP-SEG is comparable with SVM-SEG. The results demonstrate the potential of using LARS from UAVs in agriculture applications. The results are encouraging. Analysis was carried out on single images in this work. This work can be extended to carry out mapping of larger land area by analyzing orthomosaiced images covering larger area. Further geotagging the images will help to map the segmented images to physical locations.

\section{REFERENCES}

[1]Li, W., MacBean, N., Ciais, P., Defourny, P., Lamarche, C., Bontemps, S., Houghton, R.A. and Peng, S., 2018. Gross and net land cover changes in the main plant functional types derived from the annual ESA CCI land cover maps (1992-2015). Earth System Science Data, 10(1), p.219. 
[2]Costa, H., Foody, G.M. and Boyd, D.S., 2018. Supervised methods of image segmentation accuracy assessment in land cover mapping. Remote Sensing of Environment, 205 , pp.338-351.

[3] Ayele, G.T., Tebeje, A.K., Demissie, S.S., Belete, M.A., Jemberrie, M.A., Teshome, W.M., Mengistu, D.T. and Teshale, E.Z., 2018. Time Series Land Cover Mapping and Change Detection Analysis Using Geographic Information System and Remote Sensing, Northern Ethiopia. Air, Soil and Water Research, 11, p.1178622117751603.

[4] Saberioon, M.M., Amin, M.S.M., Anuar, A.R., Gholizadeh, A., Wayayok, A. and Khairunniza-Bejo, S., 2014. Assessment of rice leaf chlorophyll content using visible bands at different growth stages at both the leaf and canopy scale. International Journal of Applied Earth Observation and Geoinformation, 32, pp.35-45.

[5] Ramesh K.N, Meenavathi M.B Agriculture Crop Area mapping in images acquired using Low Altitude Remote Sensing. International Journal of Computer Sciences and Engineering 6.1 (2018): 55-62.

[6] Hassanein, Mohamed; Lari, Zahra; El-Sheimy, Naser. 2018. "A New Vegetation Segmentation Approach for Cropped Fields Based on Threshold Detection from Hue Histograms." Sensors 18, no. 4: 1253.

[7] Torres-Sánchez, J., Peña, J.M., De Castro, A.I. and LópezGranados, F., 2014. Multi-temporal mapping of the vegetation fraction in early-season wheat fields using images from UAV. Computers and Electronics in Agriculture, 103, pp.104-113.
[8] Guerrero, J.M., Pajares, G., Montalvo, M., Romeo, J. and Guijarro, M., 2012. Support vector machines for crop/weeds identification in maize fields. Expert Systems with Applications, 39(12), pp.11149-11155.

[9] Tellaeche, A., Pajares, G., Burgos-Artizzu, X.P. and Ribeiro, A., 2011. A computer vision approach for weeds identification through Support Vector Machines. Applied Soft Computing, 11(1), pp.908-915.

[10]Guerrero, J.M., Pajares, G., Montalvo, M., Romeo, J. and Guijarro, M., 2012. Support vector machines for crop/weeds identification in maize fields. Expert Systems with Applications, 39(12), pp.11149-11155.

[11] Mountrakis, G., Im, J. and Ogole, C., 2011. Support vector machines in remote sensing: A review. ISPRS Journal of Photogrammetry and Remote Sensing, 66(3), pp.247-259.

[12]Orhan, U., Hekim, M. and Ozer, M., 2011. EEG signals classification using the K-means clustering and a multilayer perceptron neural network model. Expert Systems with Applications, 38(10), pp.13475-13481.

[13]Otsu, N., 1979. A threshold selection method from graylevel histograms. IEEE transactions on systems, man, and cybernetics, 9(1), pp.62-66.

[14] Bazi, Y. and Melgani, F., 2006. Toward an optimal SVM classification system for hyperspectral remote sensing images. IEEE Transactions on Geoscience and Remote Sensing, 44(11), pp.3374-3385. 